УДК 517.9

\title{
Fractional order theory in a semiconductor medium photogenerated by a focused laser beam
}

\author{
F.S. Alzahrani ${ }^{1}$, I.A. Abbas ${ }^{1,2}$ \\ ${ }^{1}$ Nonlinear Analysis and Applied Mathematics Research Group, Department of Mathematics, King Abdulaziz University, \\ Jeddah, 21589, Saudi Arabia \\ ${ }^{2}$ Department of Mathematics, Faculty of Science, Sohag University, Sohag, 82524, Egypt
}

\begin{abstract}
In this paper, the fractional order theory has been applied for thermal, elastic and plasma waves to determine the carrier density, displacement, temperature and stress in a semiconductor medium. The thermal, elastic and plasma waves in a semi-infinite medium photogenerated by a focused laser beam were analyzed. The Laplace transformation is used to express the governing equation and solved analytically by applying eigenvalue approach methodology in that domain. A semiconducting material like as silicon was considered. According to the numerical results and graphics, the fractional order parameter and thermal relaxation time may play an important role in the behavior of all physical quantities.
\end{abstract}

Keywords: fractional order theory, photothermoelastic waves, eigenvalue approach, laser beam, Laplace transform

DOI 10.24411/1683-805X-2018-11004

\section{Дробное исчисление при описании полупроводника под воздействием сфокусированного лазерного луча}

\author{
F.S. Alzahrani ${ }^{1}$, I.A. Abbas ${ }^{1,2}$ \\ ${ }^{1}$ Университет короля Абдулазиза, Джидда, 21589, Саудовская Аравия \\ ${ }^{2}$ Сохагский университет, Сохаг, 82524, Египет
}

\begin{abstract}
С использованием дробного исчисления для описания тепловых, упругих и плазменных волн определены концентрация носителей заряда, смещения, температура и напряжения в материале полупроводника. Проведен анализ распространения тепловых, плазменных и упругих волн в полубесконечной среде при воздействии сфокусированного лазерного луча. Методом преобразования Лапласа найдено определяющее соотношение, которое решено аналитически на основе собственного значения в данной области. Исследование проведено для материала полупроводника со свойствами кремния. Результаты исследования показали, что дробный параметр порядка и время тепловой релаксации могут сильно влиять на изменение всех физических величин.

Ключевые слова: дробное исчисление, фототермоупругие волны, подход на основе собственных значений, лазерный луч, преобразование Лапласа
\end{abstract}

\section{Introduction}

When a semiconductor with band gap energy $E_{\mathrm{g}}$ is illuminated by a laser beam with an energy $E$ higher than $E_{\mathrm{g}}$, an excitation process of electrons will take place. The excited electrons will transfer to an energy level from the valence band with energy of $E-E_{\mathrm{g}}$ above the conduction band edge. Then these photoexcited free carriers will relaxe to one of the unfilled levels nearby the conduction band bottom (transition of nonradiative). After relaxation there are electron and hole plasma which is followed by the formation of hole-electron pairs through the recombination process. In semiconductors there is a periodic elastic deformation produced by the photoexcited carriers known as electronic deformation. The electronic deformation may cause local tensions in the sample which can introduce plasma waves which are similar to the heat wave generated by local periodic elastic deformation. Considerable attention has been currently given to the surface waves of bounded plasma systems. The existence of plasma boundaries makes it possible for various surface wave modes to arise, which may in some cases have frequency spectra drastically different from those of volume-wave modes. 
Understanding of transport phenomena in the solid through the development of spatially in situ resolved probes has great of attention. In the present work we try to measuring transport processes based on the principle of optical beam deflection using a photothermal approach that can be considered as an expansion of the photothermal deflection technique. Such a technique is contactless and directly yields the parameters of electronic and thermal transport at the semiconductor surface or at the interface and within the bulk of the semiconductor. Pure silicon is an intrinsic semiconductor and is used widely in semiconductor industry, for example, the monocrystalline $\mathrm{Si}$ is used to produce silicon wafers. In general, the conduction in the semiconductor (pure $\mathrm{Si}$ ) is not the same as in metals. Both the electrons and holes are responsible about the conduction in semiconductors as well as the electrons that may be released from atoms by heat. Therefore electric resistance of semiconductors decreases with temperature increasing.

Todorovic et al. [1-3] discussed theoretical and experimental results on microelectromechanical structures in plasma, thermal and elastic waves. These results give valuable information about carrier recombination and transport mechanisms in semiconductors. Also, the study includes the variations in propagating both plasma and thermal waves due to the linear coupling between heat and mass transport (i.e., thermos diffusion). The effects of thermoelastic and electronic deformations in semiconductors without considering the coupled system of the equations of thermal, elastic and plasma waves have been studied [4-6]. In addition, ocal thermoelastic deformations at the sample surface due to the excitation by a probe beam have been analyzed by Rosencwaig et al. [7], then Opsal and Rosencwaig [8] study the depth profiling of thermal and plasma waves in silicon. On the other hand, Song et al. [9, 10] study in detail the generalized thermoelastic vibrations due to optically excited semiconducting microcantilevers. They concluded that the plane wave reflection in a semiconducting material is under the theory of generalized thermoelasticity and photothermal theory $[11,12]$.

Many existing models of physical processes have been modified successfully by using the fractional calculus. Fractional order of weak, normal and strong heat conductivity under generalized thermoelastic theory were established by Youssef $[13,14]$ who developed the corresponding variational theorem also. The theory was then used to solve the $2 \mathrm{D}$ problem of thermal shock using the Laplace and Fourier transforms [15]. Based on a Taylor expansion of fractional order of time, a new model of fractional heat equation was established by Ezzatt and Karamany [16-18] and Sherief et al. [19]. Sherief and Abd El-Latief [20] studied the effect of the fractional order parameter and the variable thermal conductivity on a thermoelastic half-space. Due to thermal source, the effect of fractional order parameter on plane deformation in a thermoelastic medium was studied by Kumar et al. [21]. Recently, Abbas [22-25] stud- ied the effect of fractional order on thermoelastic problems by using the eigenvalue approach.

The present work is an attempt to get a new picture of photothermoelastic theory with one relaxation time using the fractional calculus theories. Based on the eigenvalue approach and Laplace transformation, the governing nonhomogeneous equations are processed using an analyticalnumerical technique. From the numerical results, the physical interpretations are given for the distribution of physical quantities observed in this study.

\section{Formulation of the problem}

A homogeneous semiconducting material is considered. The theoretical analysis of the transport processes in a semiconductor material involved in the study of coupled thermal, plasma and elastic waves simultaneously. The main variables are the distribution of temperature $T(\mathbf{r}, t)$, the density of carriers $n(\mathbf{r}, t)$ and the elastic displacement components $u_{i}(\mathbf{r}, t)$. When an ultrafast laser $Q(\mathbf{r}, t)$ falls on an isotropic, elastic and homogeneous semiconductor, the governing equations of motion, plasma and heat conduction under fractional order theory can be described as $[2,26-$ 29]:

$$
\begin{aligned}
& \mu\left(u_{i, j j}+u_{j, i j}\right)+\lambda u_{j, i j}-\gamma_{n} N_{, i}-\gamma_{t} \Theta_{, i}=\rho \frac{\partial^{2} u_{i}}{\partial t^{2}}, \\
& D_{\mathrm{e}} N_{, j j}=\frac{\partial N}{\partial t}+\frac{N}{\tau}-\delta \frac{\Theta}{\tau}-Q(\mathbf{r}, t), \\
& K \Theta_{, j j}=-\frac{E_{\mathrm{g}}}{\tau} N+\left(1+\frac{\tau_{0}^{v}}{\Gamma(\gamma+1)} \frac{\partial^{v}}{\partial t^{\nu}}\right) \times \\
& \times\left(\rho c_{\mathrm{e}} \frac{\partial \Theta}{\partial t}+\gamma_{\mathrm{t}} T_{0} \frac{\partial u_{j, j}}{\partial t}-\delta_{E} Q(\mathbf{r}, t)\right), 0<v \leq 1,
\end{aligned}
$$

the stress-strain relations are expressed as

$$
\sigma_{i j}=\mu\left(u_{i, j}+u_{j, i}\right)+\left(\lambda u_{k, k}-\gamma_{n} N-\gamma_{\mathrm{t}} \Theta\right) \delta_{i j},
$$

where $\rho$ is the medium density, $\tau_{0}$ is the thermal relaxation time (for semiconductor $t_{0}=10^{-12}-10^{-10} \mathrm{~s}$ ), $N=n-n_{0}$, $n_{0}$ is the equilibrium carrier concentration, $\Theta=T-T_{0}, T_{0}$ is the reference temperature, $u_{i}$ are the displacement components, $\lambda, \mu$ are the Lame's constants, $\gamma_{n}=(3 \lambda+2 \mu) d_{n}$, $d_{n}$ is the electronic deformation coefficient, $\gamma_{\mathrm{t}}=(3 \lambda+$ $+2 \mu) \alpha_{t}, \alpha_{t}$ is the coefficient of linear thermal expansion, $\sigma_{i j}$ are the stress components, $K$ is the thermal conductivity, $D_{\mathrm{e}}$ is the carrier diffusion coefficient, $\delta_{E}=E-E_{\mathrm{g}}$, $E$ is the excitation energy, $\tau$ is the photogenerated carrier lifetime, and $\delta=\partial n_{0} / \partial \Theta$ is the coupling parameter of thermal activation [30], $c_{\mathrm{e}}$ is the specific heat at constant strain, $E_{\mathrm{g}}$ is the semiconductor energy gap, $Q(\mathbf{r}, t)=\alpha \Phi(\mathbf{r}) f(t)$, $\alpha$ is the optical absorption coefficient, $\Phi(\mathbf{r})$ is the incident laser influence, $f(t)$ is the function of temporal modulation of the intensity of laser beam, $t$ is the time, and $\mathbf{r}$ is the position vector. The different parameter values with a wide range $0<v \leq 1$ cover both conductivity, $v=1$ for normal 
conductivity and $0<v<1$ for low conductivity. Let's consider an isotropic, homogeneous, semiconductor medium, occupying the region $x \geq 0$ where all the state functions depend only on the time $t$ and the variable $x$. The $x$ axis is taken perpendicular to the bounding plane of the halfspace pointing inwards. Therefore, the above equations may be take the form:

$$
\begin{gathered}
(\lambda+2 \mu) \frac{\partial^{2} u}{\partial x^{2}}-\gamma_{n} \frac{\partial N}{\partial x}-\gamma_{\mathrm{t}} \frac{\partial \Theta}{\partial x}=\rho \frac{\partial^{2} u}{\partial t^{2}} \\
D_{\mathrm{e}} \frac{\partial^{2} N}{\partial x^{2}}-\frac{N}{\tau}-\delta \frac{\Theta}{\tau}-\frac{\partial u}{\partial x}+Q(x, t)=\frac{\partial N}{\partial t} \\
K \frac{\partial^{2} \Theta}{\partial x^{2}}=-\frac{E_{\mathrm{g}}}{\tau} N+\left(1+\frac{\tau_{0}^{v}}{\Gamma(\gamma+1)} \frac{\partial^{\nu}}{\partial t^{v}}\right) \times \\
\times\left(\rho c_{\mathrm{e}} \frac{\partial \Theta}{\partial t}+\gamma_{\mathrm{t}} T_{0} \frac{\partial^{2} u}{\partial t \partial x}-\delta_{E} Q(x, t)\right) \\
\sigma_{x x}=(\lambda+2 \mu) \frac{\partial u}{\partial x}-\gamma_{n} N-\gamma_{\mathrm{t}} \Theta .
\end{gathered}
$$

\section{Initial and boundary conditions}

During recombination and transport processes (bulk and surface) of the photogenerated carriers at the surface where $x=0$ which is constrained so, the boundary conditions for the carrier density, heat flux and displacement can be written as

$$
\begin{aligned}
& D_{\mathrm{e}} \frac{\partial N}{\partial x}=s_{0} N \text { for } x=0, \\
& -K \frac{\partial \Theta}{\partial x}=E_{\mathrm{g}} s_{0} N \text { for } x=0, \\
& u(0, t)=0 \text { for } x=0,
\end{aligned}
$$

where $s_{0}$ is the speed of surface recombination and the state of the medium is initially quiescent, i.e.

$$
\begin{aligned}
& N(x, 0)=\frac{\partial N(x, 0)}{\partial t}=0, \\
& \Theta(x, 0)=\frac{\partial \Theta(x, 0)}{\partial t}=0, \\
& u(x, 0)=\frac{\partial u(x, 0)}{\partial t}=0 .
\end{aligned}
$$

For convenience, let's introduce the variables in the nondimensional form as:

$$
\begin{aligned}
& \Theta^{*}=\frac{\Theta}{T_{0}},\left(x^{*}, u^{*}\right)=\eta c(x, u), \\
& \left(t^{*}, \tau^{*}, \tau_{0}^{*}\right)=\eta c^{2}\left(t, \tau, \tau_{0}\right), \\
& N^{*}=\frac{N}{n_{0}}, \sigma_{x x}^{*}=\frac{\sigma_{x x}}{\lambda+2 \mu}, Q^{*}=\frac{Q}{n_{0} \eta^{2} c^{2} D_{\mathrm{e}}},
\end{aligned}
$$

where $c^{2}=(\lambda+2 \mu) / \rho, \mu=\rho c_{\mathrm{e}} / K$.

In terms of these nondimensional form of variables in (13), the Eqs. (5)-(12) can be written in the following forms (for convenience, the asterisks have been dropped)

$$
\frac{\partial^{2} u}{\partial t^{2}}=\frac{\partial^{2} u}{\partial x^{2}}-m_{1} \frac{\partial N}{\partial x}-m_{2} \frac{\partial \Theta}{\partial x}
$$

$$
\begin{aligned}
& m_{3} \frac{\partial N}{\partial t}=\frac{\partial^{2} N}{\partial x^{2}}-m_{3} \frac{N}{\tau}+\beta \frac{\Theta}{\tau}+Q, \\
& \frac{\partial^{2} \Theta}{\partial x^{2}}=-m_{4} \frac{N}{\tau}+\left(1+\frac{\tau_{0}^{v}}{\Gamma(v+1)} \frac{\partial^{v}}{\partial t^{\nu}}\right) \times \\
& \quad \times\left(\frac{\partial \Theta}{\partial t}+m_{5} \frac{\partial^{2} u}{\partial t \partial x}-m_{6} Q\right), \\
& \sigma_{x x}=\frac{\partial u}{\partial x}-m_{1} N-m_{2} \Theta, \\
& u=0, \frac{\partial N}{\partial x}-m_{7} N=0, \frac{\partial \Theta}{\partial x}+m_{8} N=0 \text { for } x=0, \\
& u(x, 0)=\frac{\partial u(x, 0)}{\partial t}=0, N(x, 0)=\frac{\partial N(x, 0)}{\partial t}=0, \\
& \Theta(x, 0)=\frac{\partial \Theta(x, 0)}{\partial t}=0,
\end{aligned}
$$

where

$$
\begin{aligned}
& m_{1}=\frac{n_{0} \gamma_{n}}{\lambda+2 \mu}, m_{2}=\frac{T_{0} \gamma_{\mathrm{t}}}{\lambda+2 \mu}, m_{3}=\frac{1}{\eta D_{\mathrm{e}}}, \\
& \beta=\frac{\delta T_{0}}{n_{0} \eta D_{\mathrm{e}}}, m_{4}=\frac{n_{0} E_{\mathrm{g}}}{\rho c_{\mathrm{e}} T_{0}}, m_{5}=\frac{\gamma_{\mathrm{t}}}{\rho c_{\mathrm{e}}}, \\
& m_{6}=\frac{\delta_{E} n_{0} D_{\mathrm{e}}}{K T_{0}}, m_{7}=\frac{1}{\rho c D_{\mathrm{e}}}, m_{8}=\frac{E_{\mathrm{g}} n_{0}}{\eta c K T_{0}} .
\end{aligned}
$$

For the laser pulse, the temporal profile is non-Gaussian, which can be expressed as:

$$
f(t)=\frac{I_{0} t}{t_{\mathrm{p}}^{2}} e^{-t / t_{\mathrm{p}}} .
$$

According to [9], the laser source $Q(x, t)$ can be described as

$$
Q(x, t)=\frac{I_{0} \alpha(1-R)}{2 E} \frac{t}{t_{\mathrm{p}}^{2}} e^{-\alpha x-t / t_{\mathrm{p}}},
$$

where $I_{0}$ is the energy absorbed, $R$ is the reflectivity of the sample surface, $t_{\mathrm{p}}$ is the pulse rise time. Let us define the Laplace transformation for a function $G(x, t)$ as

$$
L[G(x, t)]=\bar{G}(x, s)=\int_{0}^{\infty} G(x, t) e^{-s t} \mathrm{~d} t, s>0 .
$$

Then for both sides Eqs. (14)-(18) using the initial conditions (19), we obtain

$$
\begin{aligned}
& \frac{\mathrm{d}^{2} \bar{u}}{\mathrm{~d} x^{2}}-m_{1} \frac{\mathrm{d} \bar{N}}{\mathrm{~d} x}-m_{2} \frac{\mathrm{d} \bar{T}}{\mathrm{~d} x}=s^{2} \bar{u}, \\
& \frac{\mathrm{d}^{2} \bar{N}}{\mathrm{~d} x^{2}}-m_{3} \frac{\bar{N}}{\tau}+\beta \frac{\bar{\Theta}}{\tau}+\frac{I_{0} \alpha(1-R)}{2 E\left(s t_{\mathrm{p}}+1\right)^{2}} e^{-\alpha x}= \\
& =s m_{3} \bar{N}, \\
& \frac{\mathrm{d}^{2} \bar{\Theta}}{\mathrm{d} x^{2}}=-m_{4} \frac{\bar{N}}{\tau}+\left(1+\frac{s^{v} \tau_{0}^{v}}{\Gamma(v+1)}\right) \times \\
& \times\left(s \bar{\Theta}+s m_{5} \frac{\mathrm{d} \bar{u}}{\mathrm{~d} x}-m_{6} \frac{I_{0} \alpha(1-R)}{2 E\left(s t_{\mathrm{p}}+1\right)^{2}} e^{-\alpha x}\right),
\end{aligned}
$$




$$
\begin{aligned}
& \bar{\sigma}_{x x}=\frac{\mathrm{d} \bar{u}}{\mathrm{~d} x}-m_{1} \bar{N}-m_{2} \bar{\Theta}, \\
& \frac{\mathrm{d} \bar{N}}{\mathrm{~d} x}-c_{7} \bar{N}=0, \frac{\mathrm{d} \bar{\Theta}}{\mathrm{d} x}+c_{8} \bar{N}=0, \bar{u}=0 \text { for } x=0 .
\end{aligned}
$$

Using the eigenvalue approach, the nonhomogeneous coupled differential equations (23)-(25) can be solved, by writing them in the following vector-matrix differential equation form:

$$
\frac{\mathrm{d} V}{\mathrm{~d} x}=B V-f e^{-\alpha x}
$$

where

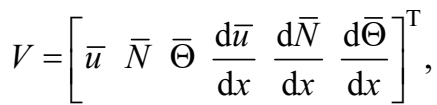

$$
\begin{aligned}
& f=\left[\begin{array}{c}
0 \\
0 \\
0 \\
0 \\
m_{9} \\
m_{10}
\end{array}\right], B=\left[\begin{array}{cccccc}
0 & 0 & 0 & 1 & 0 & 0 \\
0 & 0 & 0 & 0 & 1 & 0 \\
0 & 0 & 0 & 0 & 0 & 1 \\
b_{41} & 0 & 0 & 0 & b_{45} & b_{46} \\
0 & b_{52} & b_{53} & 0 & 0 & 0 \\
0 & b_{62} & b_{63} & b_{64} & 0 & 0
\end{array}\right]
\end{aligned}
$$

with

$$
\begin{aligned}
& m_{9}=\frac{I_{0} \alpha(1-R)}{2 E\left(s t_{\mathrm{p}}+1\right)^{2}}, m_{10}=\left(1+\frac{s^{v} \tau_{0}^{v}}{\Gamma(v+1)}\right) m_{6} m_{9}, \\
& b_{41}=s^{2}, b_{45}=m_{1}, b_{46}=m_{2}, \\
& b_{52}=m_{3}\left(s+\frac{1}{\tau}\right), b_{53}=\frac{\beta}{\tau}, b_{62}=-\frac{m_{4}}{\tau}, \\
& b_{63}=s\left(1+\frac{s^{v} \tau_{0}^{v}}{\Gamma(v+1)}\right), b_{64}=m_{5} b_{63} .
\end{aligned}
$$
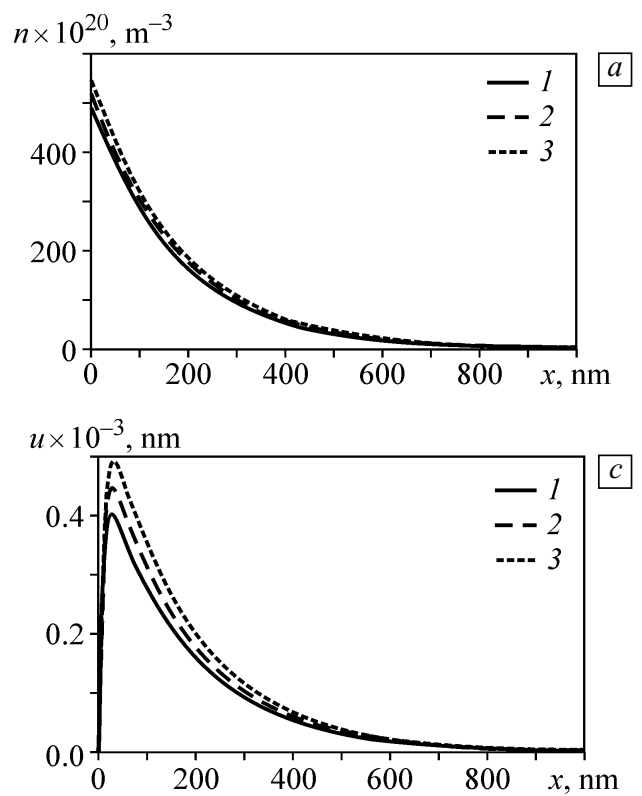

Let us apply the eigenvalue method to solve Eq. (28) [31-33]. Then, the characteristic equation of matrix $B$ can be expressed as:

$$
\chi^{6}-R_{1} \chi^{4}+R_{2} \chi^{2}+R_{3}=0,
$$

where

$$
\begin{aligned}
R_{1} & =b_{41}+b_{52}+b_{63}+b_{46} b_{64}, \\
R_{2}= & b_{41} b_{52}-b_{53} b_{62}+b_{41} b_{63}+ \\
& +b_{52} b_{63}+b_{46} b_{52} b_{64}-b_{45} b_{53} b_{64}, \\
R_{3} & =b_{41} b_{53} b_{62}-b_{41} b_{52} b_{63} .
\end{aligned}
$$

The solutions of Eq. (29) are the eigenvalues of matrix $B$ that take the form $\pm \chi_{1}, \pm \chi_{2}, \pm \chi_{3}$. Then, calculate the eigenvector $Y$ corresponding to eigenvalue $\chi$ as

$$
Y=\left[\begin{array}{c}
\chi b_{46}\left(-\chi^{2}+b_{52}\right)-\chi b_{45} b_{53} \\
-\left(\chi^{2}-b_{41}\right) b_{53} \\
-\left(\chi^{2}-b_{41}\right)\left(\chi^{2}-b_{52}\right) \\
\chi^{2} b_{46}\left(-\chi^{2}+b_{52}\right)-\chi^{2} b_{45} b_{53} \\
-\chi\left(\chi^{2}-b_{41}\right) b_{53} \\
-\chi\left(\chi^{2}-b_{41}\right)\left(\chi^{2}-b_{52}\right)
\end{array}\right] .
$$

The general solution of the nonhomogeneous equations (28) consists of the sum of the complementary solution $V_{\mathrm{c}}(x, s)$ and the particular solution $V_{\mathrm{p}}(x, s)$. The complementary solution has the following form:

$$
V_{\mathrm{c}}(x, s)=\sum_{i=1}^{3}\left(B_{i} Y_{i} e^{-\chi_{i} x}+B_{i+3} Y_{i+3} e^{\chi_{i} x}\right),
$$

where $B_{1}, B_{2}, B_{3}, B_{4}, B_{5}$ and $B_{6}$ are constants. From Eq. (31), there is the exponential function $e^{-\alpha x}$ in the inhomogeneous terms, which coincides with the exponential function in the homogeneous equation solution. Thus, the
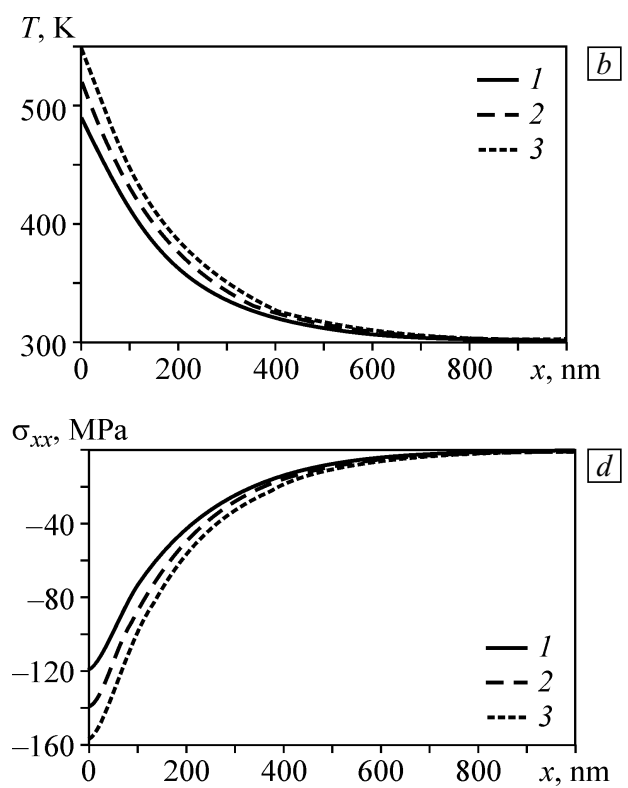

Fig. 1. The variation of carrier density $(a)$, temperature $(b)$, displacement $(c)$, stress $(d)$ with distance for $v=0.1(1), 0.5(2), 1.0(3)$ 
particular solution $V_{\mathrm{p}}(x, s)$ should be sought in the form of a quasi-polynomial vector:

$$
V_{\mathrm{p}}(x, s)=f^{*} e^{-\alpha x}
$$

where

$$
\begin{aligned}
f^{*} & =\left[f_{1}, f_{2}, f_{3}, f_{4}, f_{5}, f_{6}\right]^{\mathrm{T}}, \\
f_{1}= & \frac{1}{f_{7}}\left[\alpha b_{46}\left(b_{62} m_{9}+\left(\alpha^{2}-b_{52}\right) m_{10}\right)+\right. \\
& \left.+\alpha b_{45}\left(\left(\alpha^{2}-b_{63}\right) m_{9}+b_{53} m_{10}\right)\right], \\
f_{2}= & \frac{1}{f_{7}}\left[\left(-\left(\alpha^{2}-b_{41}\right)\left(\alpha^{2}-b_{63}\right)+\alpha^{2} b_{46} b_{64}\right) m_{9}+\right. \\
& \left.+\left(-\alpha^{2}+b_{41}\right) b_{53} m_{10}\right], \\
f_{3}= & \frac{1}{f_{7}}\left[-\left(\left(\alpha^{2}-b_{41}\right) b_{62}+\alpha^{2} b_{45} b_{64}\right) m_{9}-\right. \\
& \left.-\left(\alpha^{2}-b_{41}\right)\left(\alpha^{2}-b_{52}\right) m_{10}\right], \\
f_{4}= & -\alpha f_{1}, f_{5}=-\alpha f_{2}, f_{6}=-\alpha f_{3}, \\
f_{7}= & \alpha^{6}-R_{1} \alpha^{4}+R_{2} \alpha^{2}+R_{3} .
\end{aligned}
$$

Thus, the general solutions of Eq. (28) $V(x, s)=V_{\mathrm{c}}(x, s)+$ $+V_{\mathrm{p}}(x, s)$ can be written by

$$
V(x, s)=\sum_{i=1}^{3} B_{i} Y_{i} e^{-\chi_{i}^{x}}+f^{*} e^{-\alpha x} \text {. }
$$

Due to the conditions of regularity of the solution, the nature exponential growth of the space variable $x$ has been discarded at infinity and the constants $B_{1}, B_{2}$ and $B_{3}$ can be calculated using the boundary conditions (27). Hence, the field variables have the general solutions with respect to $x$ and $s$ in the form:

$$
\bar{u}(x, s)=\sum_{i=1}^{3} B_{i} u_{i} e^{-\chi_{i} x}+f_{1} e^{-\alpha x},
$$
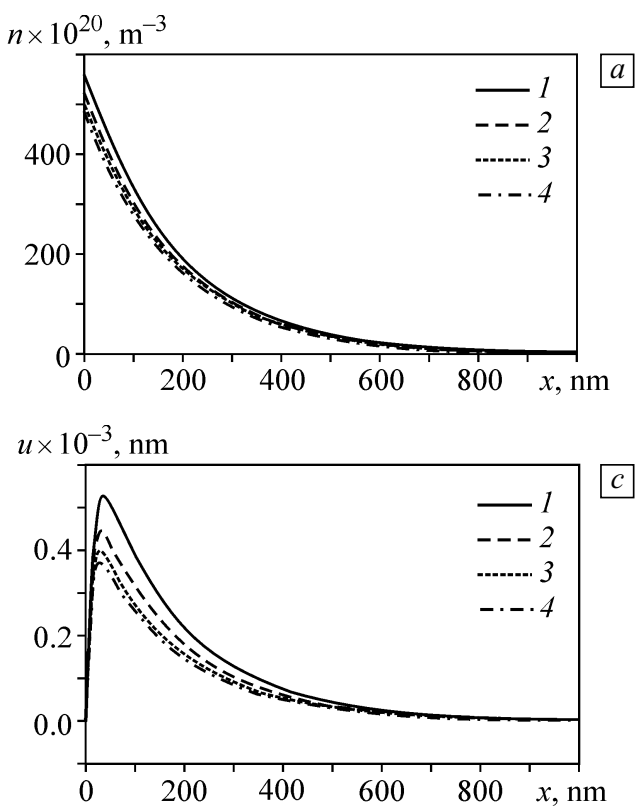

$$
\begin{aligned}
\bar{N}(x, s)= & \sum_{i=1}^{3} B_{i} N_{i} e^{-\chi_{i} x}+f_{2} e^{-\alpha x} \\
\bar{\Theta}(x, s)= & \sum_{i=1}^{3} B_{i} T_{i} e^{-\chi_{i} x}+f_{3} e^{-\alpha x} \\
\bar{\sigma}_{x x}(x, s) & =-\sum_{i=1}^{3} B_{i}\left(\chi_{i} u_{i}+m_{1} N_{i}+m_{2} T_{i}\right) e^{-\chi_{i} x}- \\
& -\left(\alpha f_{1}+m_{1} f_{2}+m_{2} f_{3}\right) e^{-\alpha x} .
\end{aligned}
$$

\section{Numerical inversions and discussion of the results}

For the general solutions of the temperature, carrier density, displacement and stress distributions, numerical inversion method was adopted. Based on Stehfest [34], the numerical inversion method was used. In this method, the inverse $G(x, t)$ of the Laplace transformation $\bar{G}(x, s)$ is approximated by the relation

$$
G(x, t)=\frac{\ln 2}{t} \sum_{j=1}^{M} V_{j} G\left(x, j \frac{\ln 2}{t}\right),
$$

where $V_{j}$ is given by the following equation:

$$
\begin{aligned}
& V_{j}=(-1)^{M / 2+1} \times \\
& \times \sum_{k=(i+1) / 2}^{\min (i, M / 2)} \frac{k^{M / 2+1}(2 k) !}{(M / 2-k) ! k !(i-k) !(2 k-1) !} .
\end{aligned}
$$

Thus, in the physical space-time domain, solutions of all variables can take the form:

$$
\begin{aligned}
& u(x, t)=\frac{\ln 2}{t} \sum_{j=1}^{M} V_{j} \bar{u}\left(x, j \frac{\ln 2}{t}\right), \\
& N(x, t)=\frac{\ln 2}{t} \sum_{j=1}^{M} V_{j} \bar{N}\left(x, j \frac{\ln 2}{t}\right),
\end{aligned}
$$
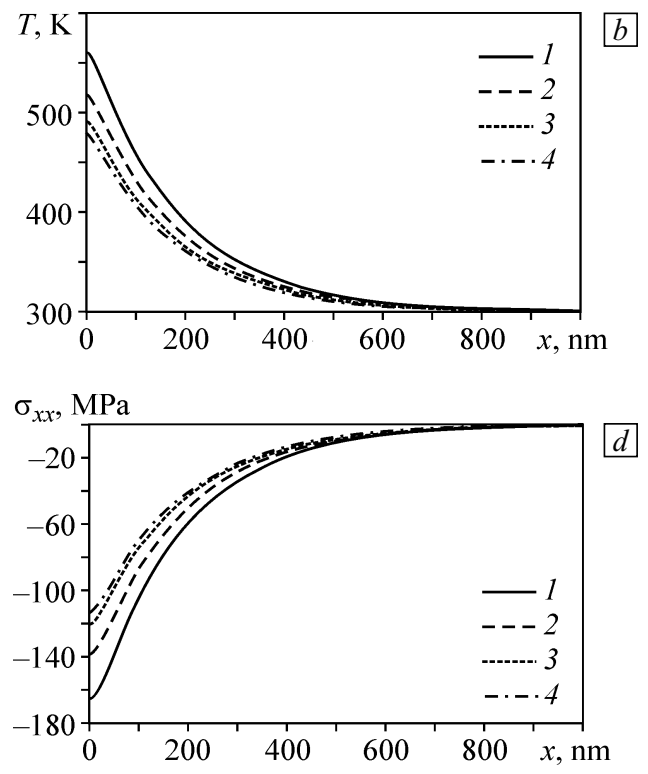

Fig. 2. The carrier density $(a)$, temperature $(b)$, displacement $(c)$ and stress distribution $(d)$ for different values of relaxation time $\tau_{0}=0$ (1), $0.1482(2), 0.7412(3), 1.4823$ (4) 


$$
\begin{aligned}
& \Theta(x, t)=\frac{\ln 2}{t} \sum_{j=1}^{M} V_{j} \bar{T}\left(x, j \frac{\ln 2}{t}\right), \\
& \sigma_{x x}(x, t)=\frac{\ln 2}{t} \sum_{j=1}^{M} V_{j} \bar{\sigma}_{x x}\left(x, j \frac{\ln 2}{t}\right) .
\end{aligned}
$$

Now, we consider a numerical example for computational purpose using a silicon-like material. The physical constants are taken as [10] $\rho=2330 \mathrm{~kg} \mathrm{~m}^{-3}, \lambda=3.64 \times 10^{10} \mathrm{~N} \mathrm{~m}^{-2}$, $\mu=5.46 \times 10^{10} \mathrm{~N} \mathrm{~m}^{-2}, E=2.33 \mathrm{eV}, E_{\mathrm{g}}=1.11 \mathrm{eV}, c_{\mathrm{e}}=$ $=695 \mathrm{~J} \mathrm{~kg}^{-1}, s_{0}=2 \mathrm{~m} \mathrm{~s}^{-1}, R=0.3, D_{\mathrm{e}}=2.5 \times 10^{-3} \mathrm{~m}^{2} \mathrm{~s}^{-1}$, $\alpha_{\mathrm{t}}=3 \times 10^{-6} \mathrm{~K}^{-1}, T_{0}=300 \mathrm{~K}, d_{n}=-9 \times 10^{-31} \mathrm{~m}^{3}, \alpha=5 \times$ $\times 10^{5} \mathrm{~m}^{-1}, I_{0}=13.4 \mathrm{~J} \mathrm{~m}^{-2}, n_{0}=10^{20} \mathrm{~m}^{-3}, \tau=5 \times 10^{-5} \mathrm{~s}$.

The described numerical techniques were used in the context of the generalized photothermal theory under the fractional order derivative. By using the relation between the variable and its nondimensional form, the variables $T$, $u, n$ and $\sigma_{x x}$ are taken in the dimensional forms and displayed graphically as in Figs. 1, 2. The calculations were performed for $t=4.447 \mathrm{ps}$.

Figures $1 \mathrm{a}-1 \mathrm{~d}$ show the variation of the temperature $T$, the displacement $u$, the carrier density $n$ and the stress $\sigma_{x x}$ with respect to the distance $x$ for different values of the fractional order parameter $v$ when $\tau_{0}=0.1$. It observed that the dotted line refer to the normal conductivity while the solid and dashed lines refer to the low conductivity. From this results, the fractional parameter $v$ has a significant effect on all the physical quantities. The effect of thermal relaxation time on the variation of the carrier density $n$, the temperature $T$, the displacement $u$ and the stress $\sigma_{x x}$ was depicted in Fig. 2. The results demonstrate the difference between the coupled photothermoelastic theory and the generalized photothermoelastic theory with one relaxation time. In this problem, the important phenomenon noticed where the medium is unbounded - the solution of any of the considered function for the generalized theory vanishes identically outside a bounded region of space. Figures $1 \mathrm{a}$ and $2 \mathrm{a}$ display the variation of carrier density along the distance $x$. It is noticed that $n$ has a highest value for $x=0$ and decreases with the increasing in distance $x$ until attaining the equilibrium carrier concentration $\left(n_{0}=\right.$ $=10^{20} \mathrm{~m}^{-3}$ ) for $x=1000 \mathrm{~nm}$. Figures $1 \mathrm{~b}$ and $2 \mathrm{~b}$ show the variation of temperature with respect to the distance $x$. It is observed that the temperature starts with its maximum value at $x=0$ and decreases gradually with increases the distance $x$ to close to the reference temperature $\left(T_{0}=300 \mathrm{~K}\right)$ beyond a wave front for the generalized photothermal theory, which satisfies our theoretical boundary conditions. Figures $1 \mathrm{c}$ and $2 \mathrm{c}$ represent the variation of displacement versus $x$. It was observed that the displacement starts from zero which satisfies the boundary condition then it reaches a peak value at a particular location proximately close to the surface and then continuously decreases to zero. Figures $1 \mathrm{~d}$ and $2 \mathrm{~d}$ show the variation of stress along the distance $x$. The magnitude of stress always starts from maxi- mum values and then decreases with distance $x$ and terminates at the zero value.

\section{Conclusion}

This work investigates the effects of fractional order and thermal relaxation time on the plasma, thermal, and elastic waves in a semiconductor medium. Analytical expressions for temperature, carrier density, displacement and stress in the medium have been obtained. Results carried out in this paper can be used to design various semiconductor elements for the coupled of plasma, thermal, and elastic waves and other fields in the materials science, physical engineering, and design of new materials to meet special engineering requirements

\section{Acknowledgments}

This work was supported by the Deanship of Scientific Research (DSR), King Abdulaziz University, Jeddah, under grant No. D-016-130-1438. The authors, therefore, gratefully acknowledge the DSR technical and financial support.

\section{References}

1. Todorovic D. Photothermal and electronic elastic effects in microelectromechanical structures // Rev. Sci. Instrument. - 2003. - V. 74(1). P. 578-581.

2. Todorovic D. Plasma, thermal, and elastic waves in semiconductors // Rev. Sci. Instrument. - 2003. - V. 74(1). - P. 582-585.

3. Song Y., Cretin B., Todorovic D.M., Vairac P. Study of photothermal vibrations of semiconductor cantilevers near the resonant frequency // J. Phys. D. Appl. Phys. - 2008. - V. 41(15). - P. 155106.

4. McDonald F.A., Wetsel G.C., Jr. Generalized theory of the photoacoustic effect // J. Appl. Phys. - 1978. - V. 49(4). - P. 2313-2322.

5. Jackson W., Amer N.M. Piezoelectric photoacoustic detection: Theory and experiment // J. Appl. Phys. - 1980. - V. 51(6). - P. 3343-3353.

6. Stearns R., Kino G. Effect of electronic strain on photoacoustic generation in silicon // Appl. Phys. Lett. - 1985. - V. 47(10). - P. 1048 1050 .

7. Rosencwaig A., Opsal J., Willenborg D.L. Thin film thickness measurements with thermal waves // Appl. Phys. Lett. - 1983. - V. 43(2). P. 166-168.

8. Opsal J., Rosencwaig A. Thermal and plasma wave depth profiling in silicon // Appl. Phys. Lett. - 1985. - V. 47(5). - P. 498-500.

9. Song Y., Todorovic D.M., Cretin B., Vairac P. Study on the generalized thermoelastic vibration of the optically excited semiconducting microcantilevers // Int. J. Solid. Struct. - 2010. - V. 47(14). - P. 18711875.

10. Song Y., Todorovic D.M., Cretin B., Vairac P., Xu J., Bai J. Bending of semiconducting cantilevers under photothermal excitation // Int. J. Thermophys. - 2014. - V. 35(2). - P. 305-319.

11. Song Y., Bai J., Ren Z. Reflection of plane waves in a semiconducting medium under photothermal theory // Int. J. Thermophys. - 2012. V. 33(7). - P. 1270-1287.

12. Song Y., Bai J., Ren Z. Study on the reflection of photothermal waves in a semiconducting medium under generalized thermoelastic theory // Acta Mech. - 2012. - V. 223(7). - P. 1545-1557.

13. Youssef H.M. Theory of fractional order generalized thermoelasticity // J. Heat Transfer. - 2010. - V. 132(6). - P. 1-7.

14. Youssef H.M., Al-Lehaibi E.A. Variational principle of fractional order generalized thermoelasticity // Appl. Math. Lett. - 2010. V. 23(10). - P. 1183-1187. 
15. Youssef H.M. Two-dimensional thermal shock problem of fractional order generalized thermoelasticity // Acta Mech. - 2012. - V. 223(6). P. $1219-1231$.

16. Ezzat M.A., El-Karamany A.S. Fractional order theory of a perfect conducting thermoelastic medium // Canad. J. Phys. - 2011. V. $89(3)$. - P. 311-318.

17. Ezzat M.A., El Karamany A.S. Theory of fractional order in electrothermoelasticity // Eur. J. Mech. A. Solid. - 2011. - P. 30(4). - P. 491500 .

18. Ezzat M.A. Theory of fractional order in generalized thermoelectric MHD // Appl. Math. Model. - 2011. - V. 35(10). - P. 4965-4978.

19. Sherief H.H., El-Sayed A.M.A., Abd El-Latief A.M. Fractional order theory of thermoelasticity // Int. J. Solid. Struct. - 2010. - V. 47(2). P. 269-275.

20. Sherief H., Abd El-Latief A.M. Effect of variable thermal conductivity on a half-space under the fractional order theory of thermoelasticity // Int. J. Mech. Sci. - 2013. - V. 74. - P. 185-189.

21. Kumar R., Gupta V., Abbas I.A. Plane deformation due to thermal source in fractional order thermoelastic media // J. Comput. Theor. Nanosci. - 2013. - V. 10(10). - P. 2520-2525.

22. Abbas I.A. Eigenvalue approach to fractional order generalized magneto-thermoelastic medium subjected to moving heat source // J. Magn. Magn. Mater. - 2015. - V. 377. - P. 452-459.

23. Abbas I.A. Generalized thermoelastic interaction in functional graded material with fractional order three-phase lag heat transfer // J. Central South Univ. - 2015. - V. 22(5). - P. 1606-1613.

24. Abbas I.A. A problem on functional graded material under fractional order theory of thermoelasticity // Theor. Appl. Fract. Mech. -2014. V. 74(1). - P. 18-22.
25. Abbas I.A. Eigenvalue approach on fractional order theory of thermoelastic diffusion problem for an infinite elastic medium with a spherical cavity // Appl. Math. Model. - 2014

26. Lord H.W., Shulman Y. A generalized dynamical theory of thermoelasticity // J. Mech. Phys. Solid. - 1967. - V. 15(5). - P. 299-309.

27. Tzou D.Y. Unified field approach for heat conduction from macro- to micro-scales // J. Heat Transfer. - 1995. - V. 117(1). - P. 8-16.

28. El-Karamany A.S., Ezzat M.A. On fractional thermoelasticity// Math. Mech. Solid. - 2011. - V. 16(3). - P. 334-346.

29. Ezzat M.A. Magneto-thermoelasticity with thermoelectric properties and fractional derivative heat transfer // Phys. B. Condens. Matter. 2011. - V. 406(1). - P. 30-35.

30. Mandelis A., Nestoros M., Christofides C. Thermoelectronic-wave coupling in laser photothermal theory of semiconductors at elevated temperatures // Optic. Eng. - 1997. - V. 36(2). - P. 459-468.

31. Das N.C., Lahiri A., Giri R.R. Eigenvalue approach to generalized thermoelasticity // Ind. J. Pure Appl. Math. - 1997. - V. 28(12). P. $1573-1594$.

32. Abbas I.A. Eigenvalue approach in a three-dimensional generalized thermoelastic interactions with temperature-dependent material properties // Comp. Math. Appl. - 2014. - V. 68(12). - P. 2036-2056.

33. Abbas I.A. A dual phase lag model on thermoelastic interaction in an infinite fiber-reinforced anisotropic medium with a circular hole // Mech. Based Design Struct. Mach. - 2015. - V. 43(4). - P. 501-513.

34. Stehfest H. Algorithm 368: Numerical inversion of Laplace transforms [D5] // Communications ACM. - 1970. - V. 13(1). - P. 47-49.

Поступила в редакцию 27.03.2017 г

Сведения об авторах

Faris S. Alzahrani, Assoc. Prof., King Abdulaziz University, Saudi Arabia, faris.kau@hotmail.com Ibrahim A. Abbas, Prof. Dr., Prof., King Abdulaziz University, Saudi Arabia, ibrabbas7@yahoo.com 